\title{
Revised element abundances for WC-type central stars
}

\author{
Helge Todt, Götz Gräfener, Wolf-Rainer Hamann \\ Institut für Physik, Universität Potsdam, Am Neuen Palais 10, 14469 Potsdam, Germany \\ email: htodt@astro.physik.uni-potsdam.de
}

\begin{abstract}
According to previous spectral analyses of Wolf-Rayet type central stars, late [WC] subtypes show systematically higher carbon-to-helium abundance ratios than early [WC] subtypes. If this were true, it would rule out that these stars form an evolutionary sequence. However, due to the different parameter domains and diagnostic lines, one might suspect systematic errors being the source of this discrepancy. In an ongoing project we are therefore checking the [WC] analyses by means of the last generation of non-LTE models for expanding stellar atmospheres which account for line-blanketing and wind clumping. So far, the abundance discrepancy is not resolved. Further element abundances $(\mathrm{H}, \mathrm{N}, \mathrm{Fe})$ are determined and compared with evolutionary predictions.
\end{abstract}

Keywords. stars: abundances, stars: mass-loss, stars: Wolf-Rayet, planetary nebulae: general

\section{Introduction}

Most central stars of planetary nebulae have a hydrogen-rich atmosphere; their optical spectra show only weak absorption lines, mainly of hydrogen and helium. In drastic contrast, there is another class of central stars whose spectra are characterized by strong emission lines of carbon, helium and oxygen. Very much like in the case of the Population I Wolf-Rayet stars, these spectra emerge from a fast and dense stellar wind. The spectral type of these hydrogen-deficient central stars is denoted as [WC], where the $\mathrm{C}$ indicates the dominance of carbon lines, while the brackets should distinguish them from their massive counterparts.

The [WC] class is divided into a sequence of subtypes, from [WC2] to [WC11] (a more recent scheme introduces also subtypes [WO1-4]). The subtypes [WC2-5] are referred to as "early" types or [WCE], while [WC6-11] subtypes are considered as "late", briefly [WCL]. [WCE] types show lines of C IV, He II and oxygen, whereas late-type spectra are dominated by C II, C III and $\mathrm{C}$ IV. Hence, the sequence of decreasing subtype number corresponds to increasing ionization, and therefore increasing effective temperature. If central stars evolve directly from the AGB to the white dwarf stage, they should first become [WCL] and then [WCE] subtypes.

\section{Hydrogen deficiency}

It is generally adopted that [WC]-type central stars have lost their hydrogen envelope in a last thermal pulse following the AGB evolution. However, it is not clear whether this last thermal pulse occurred when the star is still on the AGB (AGB Final Thermal Pulse, AFTP), or as a "Late" or "Very Late" Thermal Pulse (LTP or VLTP, respectively), which brings the star back to the beginning of the post-AGB track a second time (born-again scenario). Detailed calculations (Herwig 2001) predict that the surface composition after an LTP and VLTP resembles the former intershell abundances (see Fig. 1). In the case of the VLTP the star has almost reached the white dwarf cooling track when the last thermal pulse occurs. The envelope is then mixed downwards, and all remaining hydrogen is burnt, producing a few percent of nitrogen (Werner \& Herwig 2006). A corresponding abundance of nitrogen and the total absence of hydrogen on the stellar surface would therefore be indicative for the VLTP scenario. 


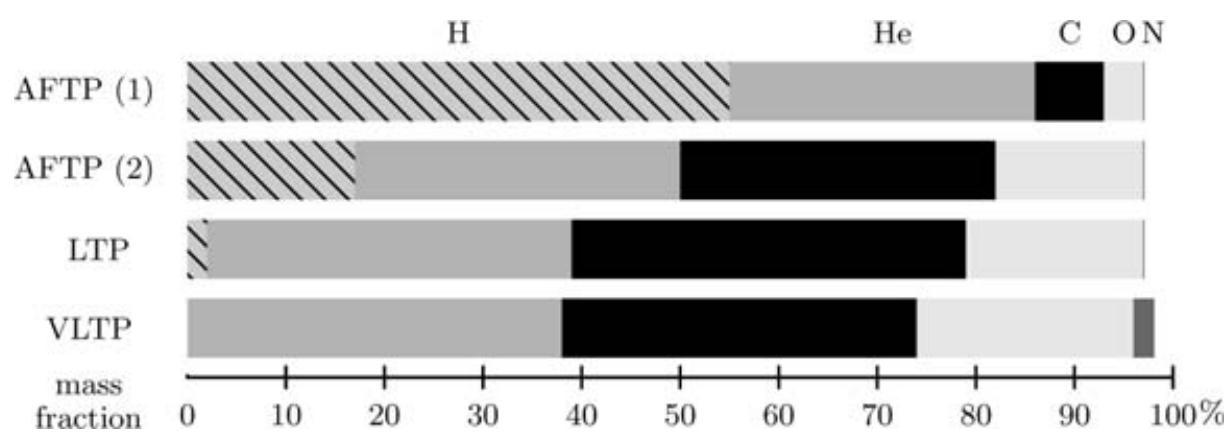

Figure 1. Stellar surface abundances predicted from stellar evolution models with simultaneous burning and mixing (Herwig 2001, Werner \& Herwig 2006). The predictions depend on the adopted scenario for the moment of the last thermal pulse; two different models are given for the AFTP case, depending on the $\mathrm{H}$ envelope mass.

\section{The Potsdam Wolf-Rayet (PoWR) model atmospheres}

Wolf-Rayet spectra form in expanding stellar atmospheres under extreme non-LTE conditions. They often have complex ionization stratifications, and different spectral lines form in different spatial regions. The hydrostatic layers are often completely hidden under the optically thick wind. Adequate model atmospheres are a prerequisite to analyze Wolf-Rayet type spectra.

The Potsdam Wolf-Rayet (PoWR) code (e.g. Hamann \& Gräfener 2004) solves the radiative transfer equation in the co-moving frame, consistently with the equations of statistical equilibrium. Iron line blanketing is included in the superlevel approach. Clumping is taken into account in the approximation of small-scale inhomogeneities, generally adopting a density contrast of $D=10$ (Hamann \& Koesterke 1998).

\section{Spectral analysis}

Distances of Galactic PNe are generally unknown, and hence the stellar parameters cannot be determined on an absolute scale. We adopt a default luminosity of $\log L / L_{\odot}=3.7$. Fortunately expanding stellar atmospheres follow a kind of scale invariance. The important parameter is the "transformed radius" $R_{\mathrm{t}}$ as a combination of mass-loss rate and stellar radius (cf. e.g. Hamann \& Gräfener 2004). The stellar mass is only of marginal influence on the spectra and adopted

Table 1. Analyses of early-type [WC] stars; $\log L / L_{\odot}=3.7, M=0.6 M_{\odot}, D=10$

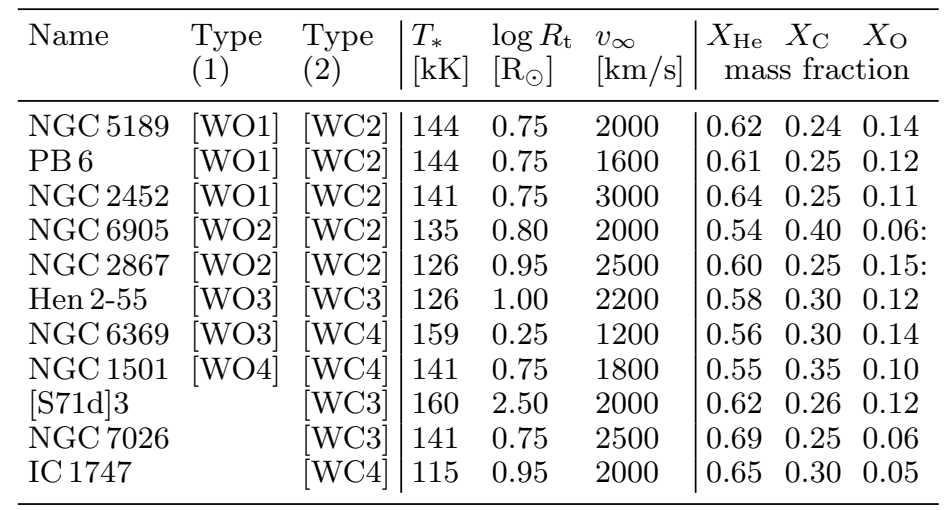

(1) new classification (Acker \& Neiner 2003) (2) old classification 


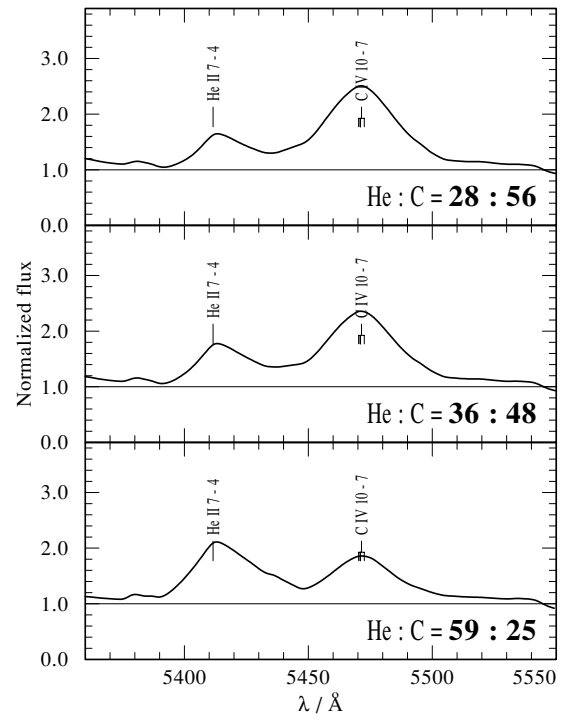

Figure 2. The pair of neighboring lines He II 5412 and CIV 5470 for models with different He: $\mathrm{C}$ mass ratios as given in the plots, while all other parameters are the same for the whole model series. Roughly equal line strength is only achieved for a He:C mass ratio of about 2:1.

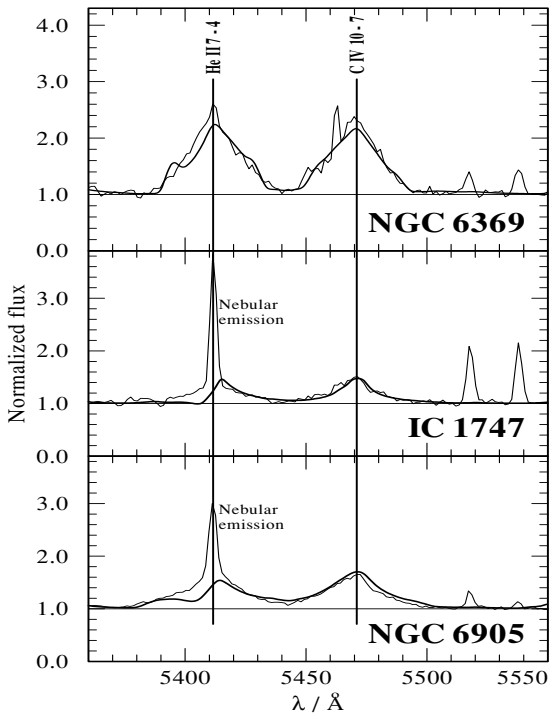

Figure 3. The diagnostic line pair HeII 5412 and Civ 5470 for three of the program stars. The two lines are generally observed to have similar strength (thin lines). Fitting models have a typical He:C mass ratio of 2:1 (thick lines). Note that the He II 5412 is often contaminated by a strong, narrow nebular emission.

to be $0.6 M_{\odot}$. Hence the free parameters to be derived by the spectral analyses are the stellar temperature $T_{*}$, the transformed radius $R_{\mathrm{t}}$, the terminal wind velocity $v_{\infty}$, and, most interesting for our purposes, the chemical composition. The results of our new analyses are compiled in Table 1. In the following we briefly discuss the chemical abundances.

Carbon and helium. In our analyses we determine the He:C ratio mainly from the line pair He II 5412 and C IV 5470 (Figs. 2 and 3). As [WCE] have highly ionized atmospheres, we checked the possible impact of very high ions of carbon and oxygen which are usually neglected in the model codes. Although the $\mathrm{C}$ IV lines are fed mainly by recombination from $\mathrm{C} v$, the inclusion of further levels above the $\mathrm{C} v$ singlet ground state did barely change the model spectra. Our new [WCE] analyses confirm in all cases He:C mass ratios of about 2:1, in contrast to the higher carbon abundance $(\mathrm{He} \approx \mathrm{C})$ found in [WCL].

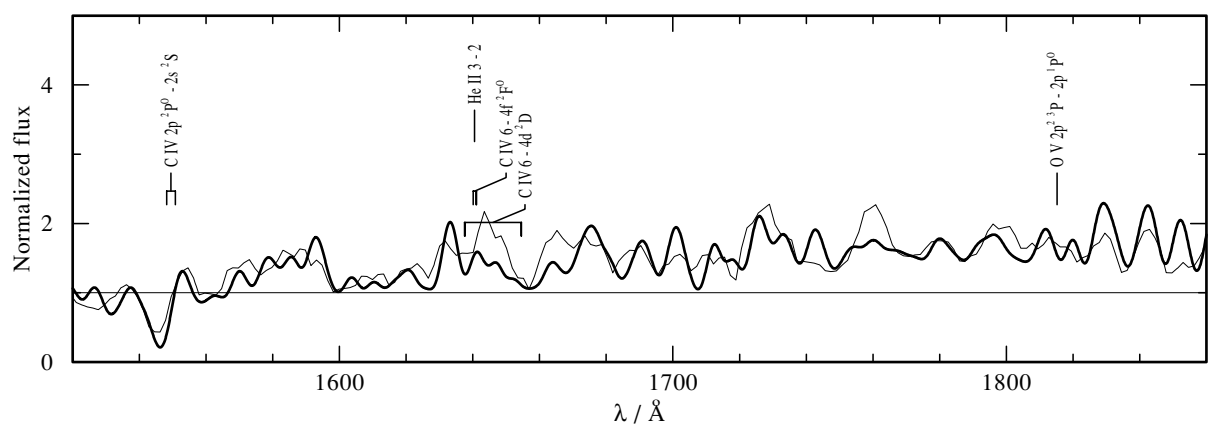

Figure 4. "Iron forest" in the late-type [WC] spectrum of $\mathrm{BD}+30^{\circ} 3639$ (thin line), compared to a model (thick line) with $X_{\mathrm{Fe}}=1.6 \cdot 10^{-3}, T=47 \mathrm{kK}, \log \left(R_{\mathrm{t}} / R_{\odot}\right)=0.8, v_{\infty}=700 \mathrm{~km} / \mathrm{s}$. 


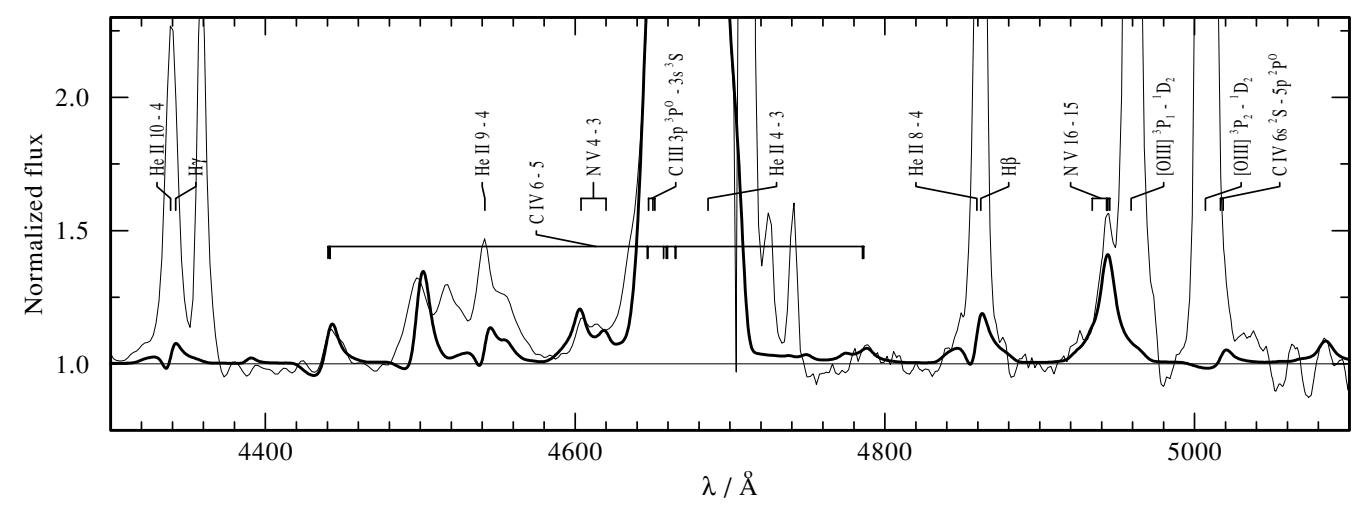

Figure 5. PB 6: Model (thick line) with $X_{\mathrm{N}}=1.5 \%$ and observation (thin line)

Nitrogen. As pointed out by Werner \& Herwig (2006), only a VLTP can efficiently produce nitrogen by $\mathrm{H}$ ingestion, leading to a $\mathrm{N}$ mass fraction of a few percent. Hence the detection of nitrogen could discriminate between the scenarios. We found indications for such overabundance in most of our analyzed [WCE] stars (except Hen 2-55 and NGC 6905), but this preliminary result must still be further substantiated.

Hydrogen. In principle $\mathrm{H}$ abundances could discriminate between the scenarios, but the $\mathrm{H}$ lines are always blended with He II and nebular emission. Small amounts of hydrogen have been found in some [WCL] stars (Koesterke 2001 and references therein), while some PG 1159 stars presumably also evolutionary related to the [WC]-type central stars - show even more hydrogen. In the hot [WCE] stars, a possible spectral signature from hydrogen would be weak. For our [WCE] program stars we can establish an upper limit of $5 \ldots 10 \%$ for the hydrogen mass fraction.

Iron. A whole "forest" of iron lines is often visible in the UV spectra of [WCL] central stars (see Fig. 4). Our sample of [WCE] stars, however, does not show detectable iron lines, neither in the observed spectra nor in the models. Therefore we cannot check the iron abundance for a possible depletion, as was observed for one cooler [WCE] star in the LMC, SMP 61 (Stasinska et al. 2004).

\section{Conclusions}

The comparison between spectral analyses and evolutionary predictions are still not conclusive. The low carbon abundance of [WCE] stars is in contrast to the [WCL] stars, and pointing to an AFTP origin. The small amount of $\mathrm{H}$ found in some [WCL] is explained from LTP models, while the possible N overabundance is rather predicted by a "Very Late Thermal Pulse (VLTP)".

\section{Acknowledgements}

We thank Adriane Liermann and Andreas Barniske for their help. This work was supported by the Bundesministerium für Bildung und Forschung (BMBF) under grant 05AVIPB/1.

\section{References}

Acker, A. \& Neiner, C. 2003, A\&BA 403, 659

Hamann, W.-R. \& Koesterke, L. 1998, A\&SA 335, 1003

Hamann, W.-R. \& Gräfener, G. 2004, A\&A 427, 697

Herwig, F. 2001, ApESSS 275, 15

Koesterke, L. 2001, ApESSS 275, 41

Stasinska, G., Gräfener, G., Peña, M., Hamann, W.-R., Koesterke, L., Szczerba R. 2004, Aछ̊A 413,329

Werner, K. \& Herwig, F. 2006, PASP 118, 183 